\title{
Sharing laughter: The humour of pre-school children with Down syndrome '
}

\author{
Vasudevi Reddy, Emma Williams and Amy Vaughan \\ Department of Psychology, University of Portsmouth, U.K.
}

\begin{abstract}
Humour and laughter have often been portrayed as fundamentally cultural and social phenomena. They can be used to tell us about children's ability to engage socially and to understand others, but have rarely been explored for this purpose. The present paper summarises the results of a study of simple forms of humour in children with Down syndrome and with autism, two groups which are reported to differ in their sociality and interpersonal understanding. Sixteen children with Down syndrome and 19 children with autism, matched on non-verbal mental age, participated in a cross-sectional study. Parental reports and video-tapes of naturalistic interaction between parents and children were analysed to show that although there were no overall differences in the presence or frequency of child or parent laughter between the two groups, there were differences in what sorts of events were more likely to prompt child laughter, the extent to which child laughter was shared, and how the children responded to others' laughter. The children with Down syndrome were more likely than the children with autism to laugh at funny faces and socially inappropriate acts and less likely to laugh in strange or inexplicable situations, and more likely to laugh at shared events. They also responded to others' laughter with attention or smiles more, and tried to re-elicit it through acts of clowning. Children with Down syndrome are thus active participants in humour and laughter, sharing it at both an emotional and a cultural level.
\end{abstract}

Keywords - Humour, Down syndrome, autism, pre-school children, laughter, sharing

\section{Introduction}

The tragic has often been seen as a unique to every individual. The first line of Anna Karenina reads, for instance, “All happy families resemble one other, each unhappy family is unhappy in its own way" (Tolstoy, 1877). In contrast, the comic has been seen as an essentially social phenomenon, existing only in society and differently so in every social group. As Eco puts it, "The tragic (and the dramatic)... are universal. The comic (is) ... bound to its time, society, cultural anthropology" (Eco, 1986). Humour and laughter have been seen as existing only through social relations and providing the ultimate platform for sociality (Bergson, 1901; Greig, 1923/1969).

If comedy is indeed a culturally sensitive accomplishment, what is it in its origins or in its expression that allows this? Can children's expressions of humour and laughter be used to understand the development of culture and sociality? Can we use expressions of humour and laughter to better

${ }^{1}$ This paper is a summary of findings reported in Reddy, Williams and Vaughan (in press) in the British Journal of Psychology. understand various developmental disabilities, in particular, of children who are believed to be advantaged in sociability and those who are believed to be impaired in this domain?

Although there is considerable research on children's responses to humorous stimuli of various kinds (see Chapman \& Foot, 1976, for a collection of papers on the topic), there is less on children's spontaneous expressions of humour or their participation in everyday, naturally occurring humour. There is even less research on either of these aspects of humour in infants and toddlers. Part of the reason for this neglect is the difficulties intrinsic to studying very young children. But in large part the neglect has been due to a theoretical assumption that humour does not really begin until the end of infancy (see, e.g. McGhee, 1979; Shultz, 1976). The memories many of us may have of children in the early school years who have grasped the idea of verbal jokes, but persist in (also rather funny) attempts to make people laugh without really understanding what it is in a joke that makes it funny, lends support to this theoretical view. However, there are simpler, non-verbal things that infants and toddlers laugh at (Sroufe \& Wunsch, 1972) and simpler forms of joking which are available to them 
(Trevarthen \& Hubley, 1978; Reddy, 1991). For instance, typically developing infants from around 8 months begin to engage in clowning (repeating acts to re-elicit laughter from others) and teasing (engaging in acts which violate newly developed shared understandings, or which provoke prohibitions from others or cause disruptions to others' ongoing actions). Acts of clowning and teasing in infancy only occur in relationships with familiar people. They may be the earliest expressions of spontaneous humour in human infants and are clearly 'social' in origin.

There is very little research on spontaneous humour in children with developmental disabilities (St. James \& TagerFlusberg, 1994, Cichetti \& Sroufe, 1976), although such research could help us better understand the nature of some disabilities better - e.g. autism, and could greatly illuminate the extent to which everyday humour may or may not play a part in the lives of children with various disabilities. The present study explored the humour and laughter of two groups of pre-school children, those with Down syndrome and those with autism, one group which anecdotal evidence suggests excels in humorous interactions and social relations and the other which we know has problems in social and communicative relations.

\section{Method}

\section{Participants}

Sixteen children with Down syndrome and nineteen children with autism were recruited through the Down Syndrome Educational Trust, the National Autistic Society and pre-schools in Hampshire. They were matched on Nonverbal Mental Age using the Cognitive Facet of the Bayley Scales of Infant Development (BSID II) with Mean mental age scores (months) of 24.4 and 24.2 respectively $(\mathrm{t}=.07$, n.s.). They were similar on the Daily Living Skills Scale of the Vineland Social Maturity (20.1 and 18.9 respectively, $t$ $=.57$, n.s.). The mean chronological ages of the two groups differed (41.3 and 49.6 months, respectively, $\mathrm{t}=2.71, \mathrm{p}<$ .03 ) as did their production vocabulary (including signs) on the MacArthur Communicative Inventory (17.9 and 11.3 months, respectively, $\mathrm{t}=2.73, \mathrm{p}<.02)$. There were nine boys and seven girls in the Down syndrome group and fifteen boys and four girls in the autism group (a gender imbalance characteristic of the disability).

\section{Procedure}

The study was a cross-sectional one, involving two home visits to the families approximately two weeks apart. In addition to the tests listed above, there were two principal modes of data collection: parental interview and videotaping of parent-child interaction. Each visit lasted approximately two hours. The Parent Interview was developed from Reddy (1991) and was used as part of a larger study on person-directed play funded by the Economic and Social Research Council (Reddy, 1998).

\section{Key Findings}

\section{Children's laughter and types of events eliciting children's laughter}

All children in both groups were reported by their parents to laugh frequently - several times a day. This reported finding was supported by analysis of children's laughter from the videotaped interactions. There was no difference between groups either in the total number of laughs (calculated as a rate per hour) or in the number of laughs started by the child (i.e. occasions where the child is the first one to laugh in an episode). However, there were some differences in what the children laughed at.

In response to an open-ended question followed by some probe questions about what sorts of things the children normally found funny or laughed at, all parents in both groups reported that children laughed at slapstick, visual events e.g. on television, and tickling and other tactile contact. However, the groups differed significantly in the extent to which children were reported to laugh at funny faces (the children with autism were reported not to laugh at this) and at socially inappropriate acts such as Mummy drinking from the baby bottle or putting the potty on her head (about $50 \%$ of the children with Down syndrome, but none of the children with autism, were reported to laugh at such things). Most interestingly, most of the parents in the autism group spontaneously mentioned that their children often laughed at things which they found odd or incomprehensible or at times when they could not understand the cause. The parents in the Down syndrome group did not report this except in one (slightly different) case, where the child was reported to laugh at 'horrible things like being smacked or watching someone get hurt'.

\section{Sharing Others' Laughter}

The frequency of adult laughter in the child's presence (from the videotaped interactions) did not differ significantly between the groups. However, the proportion of adult laughter which was directed or involved the child, did, with a greater proportion of adult laughter directed to the child in the Down syndrome group than in the autism group. The children in the two groups differed in whether they responded to adult laughter with inattention or disinterest (more in the autism group) or with brief smiles or glances even when the laugh did not involve them (more in the group with Down syndrome). This observed finding about interest in others' laughter was also reported by the parents. Most of the children in the Down syndrome group (but few in the autism group) were reported to sometimes try to join in others' laughter by laughing themselves. Several of the children with Down syndrome were reported to occasionally put on artificial laughs in order to elicit laughter or attention from others. Parental reports suggested that the reasons for putting on artificial laughs were different in the two groups of children. While the children with Down syndrome appeared to do so more often in order to join in or to get attention, the children with autism appeared to do so 'in imitation'. 


\section{Sharing their own laughter with others}

One interesting finding from the videotaped observations was the causes of laughter in the children in both groups. Laughter in the children with autism was more often prompted by 'causes' which were not shared with others occurring more often while they were alone or not in interaction with others. In the children with Down syndrome on the other hand, laughter more frequently occurred within interactions with others.

\section{Clowning: doing things to make others laugh}

The interest in others' laughter was also seen in clowning behaviour - attempts to re-elicit others' laughter by repeating acts which had previously elicited a laugh. Most of the children with Down syndrome were reported to enjoy and frequently engage in clowning. A few of the children with autism also did so, but the clowning in these few children tended to be more unclear than in the children with Down syndrome and more ritualistic and limited in variety of incidents. The children with Down syndrome were true clowns, showing an ability to make others crack up with laughter. The things they did to elicit laughter ranged from putting funny things like pants on the head, imitating the mother doing her exercises behind her back to make the sibling laugh, pretending to tickle the parent, and almost, but not quite, falling over.

\section{Teasing: violating understandings and disrupting or provoking others}

Almost all of the children with Down syndrome who engaged in clear clowning were also reported to engage in playful teasing. Teasing of three different kinds was described by parents, the most common being teasing by disrupting others' actions, followed by teasing by being non-compliant. Teasing by offering and then playfully withdrawing an object - a common early form of teasing in typically developing infants - was present but less common in the Down syndrome group and never spontaneously present in the autism group. Again, unlike the children with autism, the teasing of the children with Down syndrome tended to be of a more sophisticated kind, involving spontaneous teasing as well as teasing that was embedded in a regular family game. The incidents of teasing reported in the Down syndrome group were again much more varied and less ritualistic than in the autism group. In the autism group teasing was occasionally reported to persist or increase in the face of distress from the recipient (e.g., a younger sibling). This was never reported in the Down syndrome group, although defiance or increase in the face of parental anger was common. Reported parental attitudes to children's teasing were not different between groups: most parents reported positive feelings about teasing; there were slightly more variable (i.e. sometimes negative and sometimes positive) attitudes reported in the Down syndrome group than in the autism group.

The greater likelihood of children's teasing in the Down syndrome group was matched by a greater likelihood of parental teasing. Most of the parents in the autism group reported that they either never teased because the child would not understand it or would throw a tantrum, or did so only through simple games such as jumping out from a hiding place or peekaboo. In the Down syndrome group very few parents responded in this way. Many parents reported teasing such as mocking, deliberate mistakes such as reading the wrong story, stopping the child from getting out of the door, taking something as the child reaches for it and pretending to comply. However, the presence of reported parental teasing did not accompany greater child teasing within either the Down syndrome or the autism group. More of the children with autism were reported to respond with blankness or uncomprehending distress at parental teasing than the children with Down syndrome, but the difference was not significant.

\section{Discussion}

It is clear from the data summarised above that the contexts for early laughter and humour can be very social, but are not always so in all children. The children with Down syndrome showed a spontaneous interest in sharing their own laughter with others and in trying to elicit and share others' laughter even when it did not involve them. This suggests an access to culture in a very basic sense, which offers them the basis of enormous opportunities for further development. It was heartening to see, nonetheless, that in all children, however, whatever their disability and however severe their developmental delay, laughter was a frequent component of their everyday lives. Even when laughter does not arise from, or lead to, shared perceptions or actions, it could be seen to improve the quality of life and of the social relationships that children engage in. And because it is a feature of our lives that not only causes immediate physiological benefits but is also profoundly attractive to others, laughter has the potential of becoming shared at some point in relationships. The development of early humour and laughter appears to follow different patterns in different developmental disorders, consistent with other difficulties in social understanding and communication in each disability. Early laughter and humour, therefore, appear to be social phenomena in different ways, and however limited, offer the promise of increasing social participation and understanding in development.

\section{Correspondence}

Dr Vasudevi Reddy • Department of Psychology, University of Portsmouth, King Henry Building, King Henry I Street, Portmsouth, PO5 2DY, UK・E-mail: vasu.reddy@port.ac.uk

\section{References}

Bayley, N. (1993). Bayley Scales of Infant Development, 2nd Edition, The Psychological Corporation, San Antonio: Harcourt Brace and Company.

Bergson, H. L. (1911). Laughter: An Essay on the Meaning of the Comic. New York: Macmillan. 
Chapman, A. J. \& Foot, H. C. (1976). Humour and Laughter: Theory, Research and Applications. New Brunswick, N. J.: Transaction Publishers.

Cicchetti, D. \& Sroufe, L. A. (1976). The relationship between affective and cognitive development in Down's Syndrome infants. Child Development, 47, 920-929.

Eco, U. (1986) The Comic and the Rule. In Travels in Hyperreality. London: Pan Books.

Greig, J. Y. T. (1923/1969). The Psychology of Laughter and Comedy. New York: Cooper Square Publishers, Inc.

McGhee, P. (1979). Humour: Its Origin and Development. San Francisco: W. H. Freeman Co.

Reddy, V. (1991). Playing with others' expectations: Teasing and mucking about in the first year. In A. Whiten (Ed.), Natural Theories of Mind (pp. 143-158). Oxford: Blackwell.

Reddy, V. (1998). Person-directed play: Humour and teasing in infants and young children. Research report to the Economic and Social Research Council.

Shultz, T. R. (1976). A cognitive-developmental analysis of humour. In A. J. Chapman \& H. C. Foot (Eds.) Humour and Laughter: Theory, Research and Applications (pp. 11-36). New York: Wiley.

Sroufe, L. A., \& Wunsch, J. P. (1972). The development of laughter in the first year of life. Child Development, 43 , $1326-1344$.

St. James, P. \& Tager-Flusberg, H. (1994) An observational study of humor in children with autism and Down Syndrome. Journal of Autism and Developmental Disorders, 24(5), 603-617.

Trevarthen, C. \& Hubley, P. (1978). Secondary intersubjectivity: Confidence, confiding and acts of meaning in the first year. In A. Lock (Ed.), Action, Gesture and Symbol (pp. 183-229). London: Academic Press.

Tolstoy, L. (1877/1999) Anna Karenina. Oxford: Oxford University Press. 